\title{
Constraint production management using Fuzzy Control
}

\author{
Dr. K. Mertins, R. Albrecht and F. Duttenhofer
}

Fraunhofer-Institute for Production Systems and Design Technology (IPK)

Department Planning Technology

Pascalstr. 8-9, D-10587 Berlin, Germany

Phone ++49-30-39006-259, fax ++49-30-3911037

E-mail <frank.duttenhofer@ipk.fhg.de>

\begin{abstract}
The functions of short-time planning and control of orders on the factory's shop floor can be supported by Production Management Systems. A scheduling system capable of reflecting the planning staff's experience has been implemented. Due to the complexity of the scheduling problem and the typical lack of precision in shop expert's planning strategy descriptions a system was designed based on Fuzzy Logic.

This article presents concepts, theory and implementation of a Fuzzy Logic scheduling system for an aluminium foundry and mill.
\end{abstract}

\section{Keywords}

Production management system, order scheduling, fuzzy logic.

\section{INTRODUCTION}

Planning and control functions for the production process in manufacturing industries are supported by Production Management Systems (PMS). The operational component of a PMS has to optimize the production schedule without altering the production system or product sequence. The main objective of order scheduling as a basic subfunction of a PMS is to ensure that all products will be manufactured at minimum costs, with appropriate quality and in time. Minimum costs can be achieved in pursuing substitutional objectives like minimum inventories, high machine utilisation rates and short order throughput times [4].

Applying a phasing approach the complexity of the scheduling process can be reduced. The process can be divided into subsequent phases: coarse planning, capacity planning, order 
release, and order fine-scheduling. Order release and order fine-scheduling should be accomplished on the spot, integrating a motivated shop staff's expert knowledge and experience. By this the shortest possible reaction time can be achieved in order to cope with interrupts and delays of the manufacturing process. Shop floor control systems support these functions $[2,3]$.

\section{DECISION MAKING SUPPORTED BY FUZZY LOGIC}

The main objectives of order scheduling are the minimization of set-up expenditure and pursuing shortest lead times. Those objectives are contrary because minimizing the set-up expenditure will increase the stock on hand and the lead time of the order. For an optimal solution both targets have to be taken into consideration. Conventional methods of order scheduling will not solve the conflict because they generally aim at only one target.

A reduction of the set-up expenditure can be achieved by installing quickly acting tool change systems and performing multiple manufacturing steps in one chucking. This has been realized in metal cutting and assembly industries, whereas in metal forming industries similar efforts were made which reduced the importance of building machining sequences and allowed the concentration on lead time reduction.

In an aluminium foundry and mill the process steps include melting of aluminium, founding into bars, annealing, milling, stretching of plates and coils, and cutting. Process times vary from a few minutes to hours or days. An annealing furnace only works efficiently processing full loads, so flexibility cannot be increased reducing the lot size. The processing and therefore scheduling of optimal charges is the main planning objective. It cannot be pursued by manual order scheduling because of the complexity of order dependencies. An information system has to support the planning staff sequencing orders and collecting them to charges and machining sequences.

Nowadays also in forming industries shortest lead times have to be a planning objective in order to decrease production expenditure and delivery times and to gain access to new markets.

The ratio of set-up time to application time in the process of rolling metal alloys becomes disproportionate. Therefore an order fine-scheduling module of a shop floor control system designed for an aluminium mill [1] has to perform the additional task of building machining sequences depending on set-up state sequences. The quality of the module shows in

1. the applicability of the resulting machining sequences and in

2. the throughput time of orders.

An empirical analysis of currently practised manual planning strategies revealed that they can only partially be modelled using binary logic. Experts at the shop floor apply definite rules for order sequencing regarding one single set-up state. Their strategy for sequencing the set-up states within a planning period could only be put down unprecisely. The reasons are the conflict of the planning objectives mentioned above and the unprecise way of human communication and interaction.

Fuzzy Logic is a means for modelling problem solution strategies based on human experience, knowledge, intuition and heuristics. Humans preferably handle qualitative and not 
so often quantitative values when making decisions. Qualitative values can be modelled directly with Fuzzy Logic.

Conventional mathematical methods are often too complex or not appropriate for solving sequencing problems in a given time frame. Even when dealing with minor problems a fuzzy strategy can be applicable if exact values are not necessary or cannot be collected or used.

Adapting human problem solving strategies and fuzzy values of the variables needed in an automated planning system simplifies the documentation of the planning process and the evaluation of its results.

\section{OVERVIEW OF FUZZY LOGIC THEORY}

The following chapter presents Fuzzy Logic theory insofar as it is necessary to understand the implementation of the planning system [4].

\section{Fuzzy Sets}

Defining a precise set of values is usually done giving the exact values or the range of values. For any value it can be specified whether it belongs to a certain set or not. Another approach is to define a membership function $\mu(x)$ which specifies in binary logic whether a value $x$ belongs to the set or not [5].

In a Fuzzy Set the values of the membership function are floating point values in the range between 0 and a finite upper bound. In a normalised Fuzzy Set the upper bound equals to 1 . Fuzzy Sets can be implemented in a computer using arrays of values or defining a membership function. A function consumes less memory and allows an adjustable granularity of input values.

Classes of functions are each defined by a set of parameters, e.g. a triangle-shaped function is defined by a value $m$ with a maximal function value $\mu(m)$, by the minimum and maximum values $\mu_{\min }$ and $\mu_{\max }$ and by the distances of $m$ to both corners ( $a$ and $b$, see Fig. 1). Trapezoids and generalised LR-(left-right)-Fuzzy Sets can be defined, the latter using nonlinear functions.

To reflect terms of human communication in a computer, linguistic variables can be defined as collections of Fuzzy Sets. Linguistic variables contain common qualifying words and phrases in contrary to numerical variables.

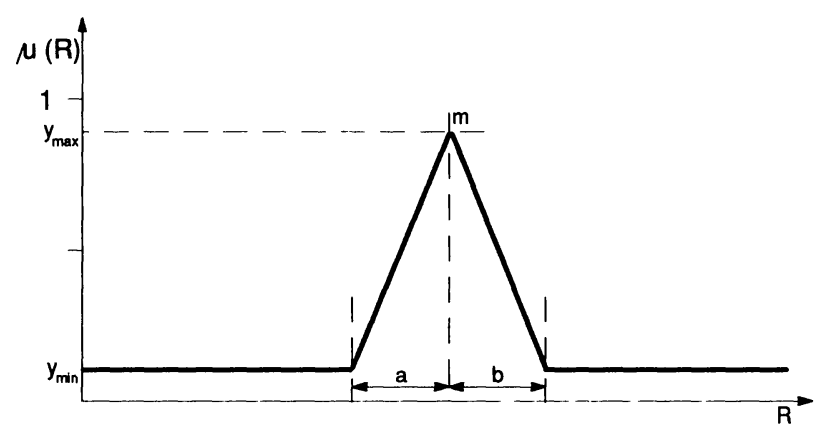

Figure 1 Triangular Fuzzy Set [5] 


\section{Operators on Fuzzy Sets}

Elementary operators on Fuzzy Sets are intersection (minimum) and union (maximum) and the unary complement. The minimum and maximum operators satisfy the commutative and the associative law which enables a recursive combination of more than two sets in any processing order. Further operators can be defined by generalising the elementary operators. A combination of minimum and maximum behaviour of an operator is feasible using parameters. Criteria for the classification of Fuzzy Set operators are e.g. associativity, commutativity, monotony, continuity, and other mathematical properties. An important non-mathematical criterion is the adaptability of an operator by parameters in order to guarantee an optimal reflection of human implication. The method of modelling human terms in operators has not yet been put on a theoretical basis and is due to the user.

\section{Fuzzy Logic}

Fuzzy Logic provides methods for fuzzy implication using Fuzzy Sets. The expert's knowledge needed for automated inferencing is implemented in "IF condition THEN conclusion" rules. The linguistic variables in those rules are combined using Fuzzy Set operators. All facts and rules are assigned a special degree of certainty. Applying the rules should lead to useful and problem solving results. Therefore the definition of Fuzzy Sets of a linguistic variable should satisfy several conditions, e.g. limitations of the degree of overlapping regions. Consistency and completeness of the rules are a precondition.

\section{SET-UP STATE SEQUENCING USING A FUZZY LOGIC APPROACH}

In Fuzzy Logic calculations, functions replace simple numbers, which leads to an increased expenditure compared to binary logic. Therefore applying Fuzzy Logic is only recommended if a solution can not (or can only with a disproportionate effort) be found applying conventional methods, as in the example application described here. Fuzzy Logic is used for the selection of the next appropriate set-up state out of a set of possible solutions. All other planning tasks are done by numeric and heuristical strategies $[4,6]$.

The determination of the sequence of set-up states is initiated by an analysis of all operations in a certain time interval. For all possible set-up states the time interval until the next set-up is calculated. The number of operations in this time interval is counted. So the mixture of orders to plan is changing with the ongoing scheduling process. The determination of the sequence of set-up states and the scheduling of operations are performed interchangeably. Only that set-up state is searched which shall be realised at the current state of planning. After that step all operations belonging to the chosen set-up state are scheduled until one of the stopping criteria matches (Fig. 2).

A Fuzzy software tool has been implemented for the calculation of an optimal set-up sequence. The way of implementation is analogous to the development of a Fuzzy Controller (Fig. 3). For the evaluation of the result of a sequencing process both time- and machinespecific criteria have to be considered.

The slack time of an order is one of the time related values. The smaller the remaining processing time the smaller the slack time may be without increasing the likelihood of a delay of the completion time. In order to be able to determine the slack time of orders with different remaining processing times the slack time has to be put in relation to the remaining processing 


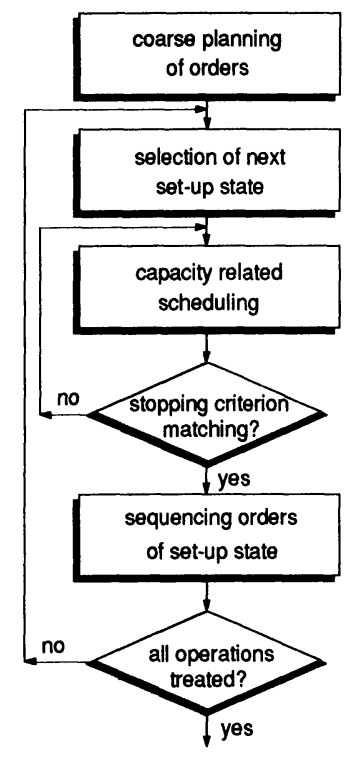

Figure 2 Process of planning [4]

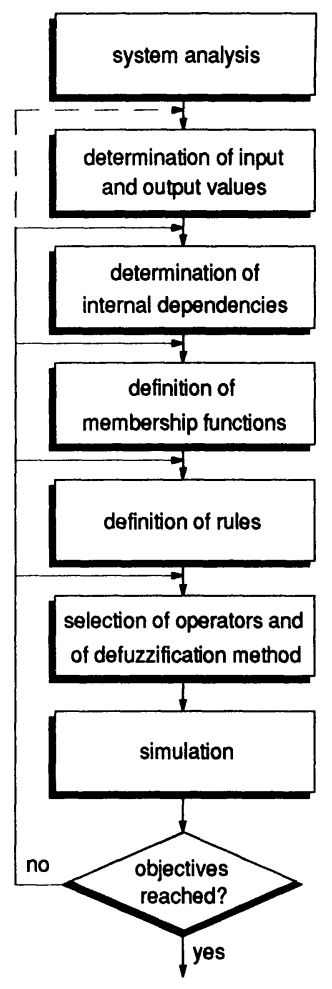

Figure 3 Development of a Fuzzy Controller [6]

time. The result is called relative slack time. Assumptions are small transportation and layingtimes in relation to processing times [7].

In case of the factory described here those conditions are not satisfied. Processing times sometimes are much shorter or even as long as the technically necessary laying-times. Therefore the relative slack time is calculated using the minimally needed remaining lead time. Transportation times are short in relation to transition times and have therefore been neglected.

The relative slack time is bound to an order, so a similar value related to set-up states has to be found which represents the relative slack time of orders in the circulation time interval. This value is calculated as follows: sum of all relative slack times of orders in the possible circulation time interval divided by the number of operations in that time interval. This value is called relative buffer.

For a roll of a mill the machine-specific value is the degree of utilization of the capacity dependent on its life time (number of planned orders). It is calculated using the number of operations with equal roll types in the circulation interval.

For the use in Fuzzy Logic all input values and the results are translated into linguistic variables. An example of Fuzzy Logic application describes the selection of the succeeding roll type of an aluminium rolling mill. The exact input parameters are transformed into linguistic 


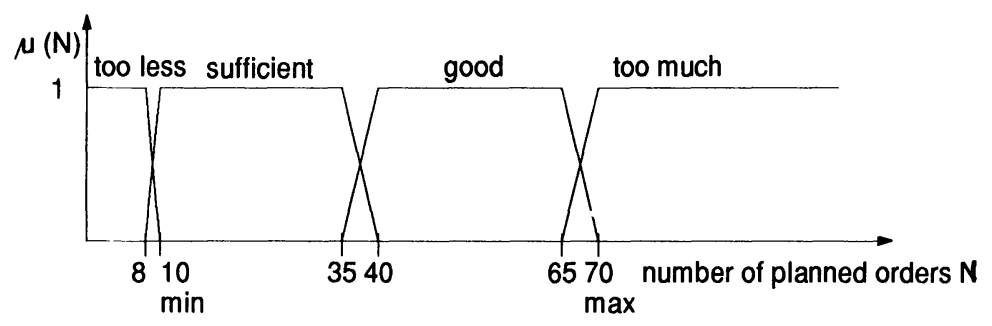

Figure 4 Membership functions of the linguistic variable 'number of planned orders' [4]

variables, as well as the output parameter which contains the decision for a specific set-up state. The membership functions of the linguistic variables are shown in Fig. 4, 5 and 6. The Fuzzy Sets are implemented in the shape of trapezoids. This shape should be used if the output of the rules shall not change throughout ranges of the input parameters, that means, a separation of linguistic values and their meanings is not possible or convenient.

The linguistic variable named number of planned orders (Fig. 4) is defined by four membership functions. The positions of the maximum values have been determined in correlation to the minimal (10) and maximal (70) number. Those values correspond to special roll types, so for each roll type separate membership functions have to be specified.

For the linguistic variable relative buffer five membership functions had to be specified (Fig. 5). The positions of the maximum values were chosen arbitrarily. Realistic values will be determined in the future along with the test of the module under production conditions. The width has been set to 1.5 shifts so far and has to be adapted to the maximum values.

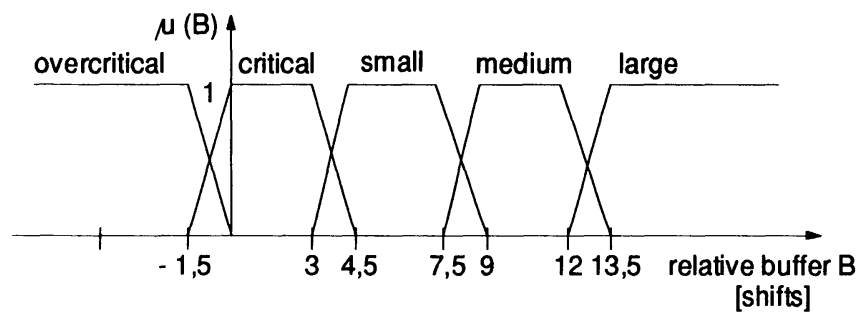

Figure 5 Membership functions of the linguistic variable 'relative buffer' [4]

The linguistic variable quality of set-up state is modelled by five membership functions (Fig. 6). The values shall indicate the quality of a set-up state in correlation to the input parameters (value in percent of quality). The positions of the maximum values are spread homogeneously, the width was set to $5 \%$.

The conclusion is based on rules of the type "IF number of planned orders is sufficient AND relative buffer is small THEN ... ". The aggregation operator determining the degree of matching the facts with the input parameters has to be realised by an AND operator. Because of its high efficiency the minimum operator was chosen. 


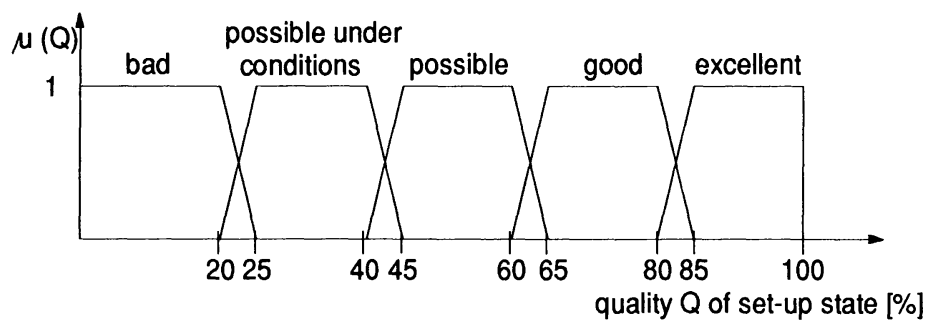

Figure 6 Membership functions of the linguistic variable 'quality of set-up state' [4]

To implement an implication operator determining the degree of validity of the conclusion the minimum operator was taken. As a consequence the minimal membership value of both input parameters (number of planned orders is sufficient and relative buffer is small) produces a value of the membership function "good" of the linguistic variable set-up state quality. No relevant factors were defined, i.e. all rules are regarded as being reliable without any restrictions. The factors equal to 1 for each rule. A necessity might arise in the future to introduce relevant factors in the model.

The single results of all rules are combined by accumulation choosing an OR operator. The result represents the final solution of the inference problem. The maximum operator was chosen because of its high efficiency.

The rules are based on the statements of experienced staff. The rules type is of the form "IF number of planned orders is sufficient AND relative buffer is small THEN quality of set-up state is good". In Table 1 all combinations are presented to form rules.

Table 1 Rules for valuation of a set-up state [4]

\begin{tabular}{|l|l|l|l|l|l|}
\multicolumn{7}{c}{} & \multicolumn{2}{c}{ number of planned orders } \\
\cline { 2 - 6 } & too less & sufficient & good & too much \\
\cline { 2 - 6 } & Overcritical & good & excellent & excellent & excellent \\
\cline { 2 - 6 } $\begin{array}{l}\text { relative } \\
\text { buffer }\end{array}$ & critical & possible & good & good & good \\
\cline { 2 - 6 } & Small & $\begin{array}{l}\text { possible } \\
\text { under } \\
\text { conditions }\end{array}$ & possible & possible & good \\
\cline { 2 - 6 } & medium & bad & $\begin{array}{l}\text { possible } \\
\text { under } \\
\text { conditions }\end{array}$ & possible & possible \\
\hline large & bad & bad & $\begin{array}{l}\text { possible } \\
\text { under } \\
\text { conditions }\end{array}$ & $\begin{array}{l}\text { possible } \\
\text { under } \\
\text { conditions. }\end{array}$ \\
\hline
\end{tabular}

Final result of the inference method is a Fuzzy Set used to evaluate a given set-up state. To be able to compare the result with other solutions it is necessary to retranslate the obtained Fuzzy Set into a concrete value (defuzzification). Methods for defuzzification are:

- division of the Fuzzy Set in two halves of the same area size and

- calculation of the centre of gravity of the resulting Fuzzy Set. 
The resulting value will be the best representation of the information contained in the input Fuzzy Sets. In the current implementation both methods are applied in order to gain further experience.

\section{INTEGRATION IN THE PRODUCTION MANAGEMENT SYSTEM}

A production planning system performs the coarse planning of all orders. The results are transferred to the scheduling system to determine the final order sequence for all machines.

The sequence can then be changed manually using disposition tools with dynamic graphics. These tools also allow an automatic recalculation of the sequence and the creation of multiple scenarios in order to be able to try different sequencing methods or test the effects of scheduling parameter manipulations.

\section{CONCLUSION}

This paper presented a set-up scheduling system based on Fuzzy Logic. Benefits for system users are easy intelligibility of the system and intelligible documentation of a complex planning process. The planning system can easily be modified in order to adapt it to changing planning conditions and strategies. Due to its high performance the system allows quick reactions on unpredictable events in the manufacturing process. Costs will be reduced by choosing the right set-up plan for most cases.

Until today only a small part of Fuzzy Logic theory is used to implement planning and control systems, despite the good prospects of the technology in the field of decision making [8]. In the near future more efficient Fuzzy systems will be realised. Some scientists stated that Fuzzy Logic will be one of the key technologies of the next century, applicable in many fields which until today have shown resistance to automation attempts.

\section{REFERENCES}

[1] Keller, A.; Mertins, K.; Albrecht, R.; Duttenhofer, F.: Auftragssteuerung für Gieß- und Walzprozesse. ZwF 87 (1992) 12, pp. 669-672

[2] Mertins, K.; Albrecht, R.; Steinberger, V.: Werkstattsteuerung - Werkstattmanagement. Wegweiser zur Einführung. Carl Hanser Verlag, München, Wien 1992

[3] Mertins, K.; Tonn, F.; Wegener, U.; Wilksch, S.: Entwicklung und industrieller Einsatz eines Fertigungsleitsystems. ZwF 87 (1992) 2, pp. 75-79

[4] Mertins, K.; Albrecht, R.; Wegener, U.; Duttenhofer, F.: Set-Up Scheduling by Fuzzy Logic. Proceedings of the Fourth International Conference on Computer Integrated Manufacturing and Automation Technology. IEEE Computer Society Press, Los Alamitos CA 1994, pp. 345-350

[5] Tilli, T.: Fuzzy-Logik: Grundlagen, Anwendungen, Hard- und Software. Franzis-Verlag, München 1991

[6] Tilli, T.: Automatisierung mit Fuzzy-Logik. Franzis-Verlag, München 1993

[7] Zimmermann, H.J.: Unscharfe Logik stößt auf großes Interesse. VDI-Nachrichten Nr. 20, 17. Mai 1991, pp. 14-15 
[8] Zimmermann, H.J.: Fuzzy-Technologien: Prinzipien, Werkzeuge, Potentiale. VDIVerlag, Düsseldorf 1993

\section{BIOGRAPHIES}

Dr.-Ing. Kai Mertins, born in 1947, has studied Control Theory in Hamburg and Economy together with Production Technology at the Technical University of Berlin. He became member of the scientific staff of the University Institute for Machine Tool and Manufacturing Technology (IWF), Berlin/FRG. Since 1983 he had been head of the department „Production Control and Manufacturing Systems“ at the Fraunhofer-Institute for Production Systems and Design Technology (IPK), Berlin/FRG, where he is Director for Planning Technology since 1988. Special field of interest: Manufacturing strategy development, planning for production systems, shop floor control and simulation.

Rolf Albrecht, born in 1950, has studied Engineering at the Technical University in Berlin and finished his studies with a diploma in 1978. Since this time he worked as a scientist for different institutions of the TU Berlin. From 1980 to 1985 he worked as an author for the scientific journal „ZwF-Zeitschrift für wissenschaftliche Fertigung“. He took part at different scientific programs at IBM Heidelberg. Since 1990 he is head of the department „Production Control" of the Fraunhofer Institute in Berlin.

Frank Duttenhofer, born in 1964, has studied Computer Science at the University of Erlangen-Nürnberg. Since 1990 he is member of the scientific staff of the Fraunhofer Institute in Berlin. He is now head of the scientific group „Manufacturing Information“. 